\title{
$\mathrm{Mn}$ 和 $\mathrm{Fe}$ 掺杂对尖晶石氧化物 $\mathrm{Co}_{2} \mathrm{MnO}_{4}$ 结构和磁性的影响
}

\author{
孟凡斌 ${ }^{1}$, 马晓帆 ${ }^{1}$, 张 炜 $^{1}$, 吴光恒 ${ }^{2}$, 张玉洁 ${ }^{1}$
}

(1. 河北工业大学 材料科学与工程学院, 天津 300130; 2. 中国科学院 物理研究所, 北京 100190)

摘 要: 以金属盐及柠檬酸为原料, 采用溶胶-凝胶法制备了尖晶石氧化物 $\mathrm{Co}_{2-x} \mathrm{Mn}_{1+x} \mathrm{O}_{4}$ 和 $\mathrm{Co}_{2-x} \mathrm{Fe}_{x} \mathrm{MnO}_{4}$ 系列, 通过 XRD、FT-IR 及 PPMS 等手段研究了 $\mathrm{Co}_{2} \mathrm{MnO}_{4}$ 及系列掺杂样品的成相、结构、磁性等特征。结果表明, $\mathrm{Co}_{2-x} \mathrm{Mn}_{1+x} \mathrm{O}_{4}$ 系列在 $x<0.6$ 时, 呈单相立方结构, 晶格常数和磁性随着 $\mathrm{Mn}$ 掺杂量的增加而增大, $x \geqslant 0.6$ 时逐渐向四方结构转化, 磁性下降, 并呈现磁化强度不易饱和的特征; $\mathrm{Co}_{2-\mathrm{x}} \mathrm{Fe}_{x} \mathrm{MnO}_{4}$ 系列样品在 $x<1.75$ 成分范围内均可保持立方结构, 且 晶格常数和磁性都随着 $x$ 增大而提高。这些变化主要是由于掺杂原子尺度及磁矩均大于原有元素, 掺杂后样品内部 的磁性相互作用有所增强。

关 键 词: 尖晶石氧化物; 晶体结构; 磁性耦合

中图分类号: TQ174 文献标识码: A

\section{Structure and Magnetic Property of Fe and Mn Doped Spinel $\mathrm{Co}_{2} \mathrm{MnO}_{4}$}

\author{
MENG Fan-Bin ${ }^{1}$, MA Xiao-Fan ${ }^{1}$, ZHANG Wei ${ }^{1}$, WU Guang-Heng ${ }^{2}$, ZHANG Yu-Jie ${ }^{1}$ \\ (1. School of Materials Science and Engineering, Hebei University of Technology, Tianjin 300130, China; 2. Institute of Phys- \\ ics, Chinese Academy of Sciences, Beijing 100190, China)
}

\begin{abstract}
Spinel solid solution $\mathrm{Co}_{2-x} \mathrm{Mn}_{1+x} \mathrm{O}_{4}$ and $\mathrm{Co}_{2-x} \mathrm{Fe}_{x} \mathrm{MnO}_{4}$ were synthesized by Sol-Gel method using metal nitrates and citric acid as the starting materials. Crystalline phases, structure and magnetic properties of the $\mathrm{Co}_{2} \mathrm{MnO}_{4}$ doped with different concentrations of Fe and Mn were investigated by XRD、FT-IR and PPMS, respectively. Results show that $\mathrm{Co}_{2-x} \mathrm{Mn}_{1+x} \mathrm{O}_{4}$ series are single-phase cubic spinel structure at $x<0.6$, the lattice parameter as well as the saturation magnetization are found to be increasing with the $\mathrm{Mn}$ content increasing. $\mathrm{Co}_{2-x} \mathrm{Mn}_{1+x} \mathrm{O}_{4}$ series transform to tetragonal structure gradually at $x \geqslant 0.6$, which result in the decline of magnetic properties and difficulty of reaching saturation. $\mathrm{Co}_{2-x} \mathrm{Fe}_{x} \mathrm{MnO}_{4}$ samples have cubic spinel structure at $x<1.75$, the lattice parameter and magnetic increase with the increase of Fe-substitution. When the Fe content increases to $1.75, \mathrm{Fe}_{2} \mathrm{O}_{3}$ phase is formed in the sample. The changes of performance are mainly due to the larger atom radius of the doping atoms ( $\mathrm{Fe}$ or $\mathrm{Mn}$ ) as compared to that of Co. And magnetic moment is another important factor to the performance change. The first-principles calculation results show that the magnetic moments of $\mathrm{Fe}$ and $\mathrm{Mn}$ are larger than that of $\mathrm{Co}$. As a result, the interatomic magnetic interaction is enhanced by doping.
\end{abstract}

Key words: spinel-type oxide; crystal structure; magnetic coupling

尖晶石型氧化物以其特殊的晶体结构、优良丰 富的物理化学性能, 得到人们的广泛关注 ${ }^{[1-4]}$ 。立方
尖晶石氧化物的通式为 $\mathrm{A}^{2+} \mathrm{B}^{3+}{ }_{2} \mathrm{O}^{2-}$, 其中氧离子构 成四面体和八面体结构, 金属离子 $\mathrm{A}^{2+}$ 和 $\mathrm{B}^{3+}$ 完全或 
者部分填充于四面体和八面体间隙 ${ }^{[5-6]}$ 。决定金属离 子所占位置的因素比较复杂, 主要是受电负性与原 子间成键的影响 ${ }^{[7-8]} 。 \mathrm{Co}_{2} \mathrm{MnO}_{4}$ 具有反尖晶石结构, $\mathrm{Co}^{2+}$ 占据四面体位置, $\mathrm{Co}^{3+}$ 与 $\mathrm{Mn}^{3+}$ 共同填充八面体 间隙 ${ }^{[9-11]}$ 。

$\mathrm{Co}_{2} \mathrm{MnO}_{4}$ 在固体氧化物电池、超级电容器和多 铁性(磁电耦合) 等领域都有应用 ${ }^{[12-14]}$ 。这类材料在 电性能方面的研究报导较多, 而从磁性方面考虑, 由于材料中部分原子占位混乱 ${ }^{[15]}$, 并且磁矩排列非 共线 ${ }^{[4]}$, 磁性能受制备方法和元素掺杂影响较大, 相关报道不多。目前, 报道较多的是用非磁性 $\mathrm{Bi}$ 原 子对 $\mathrm{Co}_{2} \mathrm{MnO}_{4}$ 进行掺杂, 改善材料的磁性能。 $\mathrm{Bi}$ 原 子本身没有磁性, 对磁性的改变主要是由于 $\mathrm{Bi}$ 的原 子半径大于 $\mathrm{Co}$ 的原子半径, 掺入后会破坏 $\mathrm{Co}_{2} \mathrm{MnO}_{4}$ 原有的氧八面体结构, 同时削弱 $\mathrm{Co}^{2+}-\mathrm{Co}^{2+}$ 的反铁磁有序性 ${ }^{[16]}$ 。本工作选取磁性原子 $\mathrm{Mn} 、 \mathrm{Fe}$ 对 $\mathrm{Co}_{2} \mathrm{MnO}_{4}$ 进行掺杂, 主要研究材料结构以及磁性 能, 通过检测样品的成相、晶体结构、磁性等变化 规律, 结合第一性原理计算, 分析尖晶石氧化物中 各类原子间的相互作用及其对宏观性能的影响。

\section{1 实验方法}

以分析纯 $\mathrm{Co}\left(\mathrm{NO}_{3}\right)_{2} \cdot 6 \mathrm{H}_{2} \mathrm{O} 、 \mathrm{Fe}\left(\mathrm{NO}_{3}\right)_{3} \cdot 9 \mathrm{H}_{2} \mathrm{O}$ 和 $\mathrm{Mn}\left(\mathrm{NO}_{3}\right)_{2}$ 溶液为原料, 按 $\mathrm{Co}_{2-x} \mathrm{Mn}_{1+x} \mathrm{O}_{4}(0 \leqslant x \leqslant$ 1)、 $\mathrm{Co}_{2-x} \mathrm{Fe}_{x} \mathrm{MnO}_{4}(0 \leqslant x \leqslant 2)$ 化学计量比溶于水, 并 使用磁力搅拌器摚拌 $1 \mathrm{~h}$, 待溶液混合均匀。按照阳 离子与柠檬酸 $1: 3$ 比例加入柠檬酸, 继续搅拌待柠 檬酸完全混合均匀后, 加入氨水调节溶液 $\mathrm{pH}$ 至 6 左右. 将混合均匀的溶液放入 $80^{\circ} \mathrm{C}$ 水浴锅中持续摚 拌, 蒸发水分, 至溶液逐渐形成凝胶。此凝胶再在 $150^{\circ} \mathrm{C}$ 下加热 $10 \mathrm{~h}$ 后冷却至室温, 然后在箱式炉中 $1000^{\circ} \mathrm{C}$ 下炦烧 $1 \mathrm{~h}$, 得到最终样品。

利用 2500PC 型 X 射线衍射仪(XRD)分析样品 的晶体结构和相组成, 使用 $\mathrm{Cu}$ 靶; 在 VECTOR22 型红外光谱仪上测量样品的红外光谱; 在美国综合 物性测量系统(PPMS)上进行样品的磁性能表征。采 用基于第一性原理计算的 Materials Studio, castep 软 件包进行理论分析。

\section{2 结果和讨论}

图 1(a)为 $\mathrm{Co}_{2-x} \mathrm{Mn}_{1+x} \mathrm{O}_{4}$ 系列样品的 $\mathrm{XRD}$ 图谱, $\mathrm{Co}_{2} \mathrm{MnO}_{4}$ 样品具有立方尖晶石结构, 没有出现杂 峰。图中标出了晶面指数, 并计算得到 $\mathrm{Co}_{2} \mathrm{MnO}_{4}$ 晶
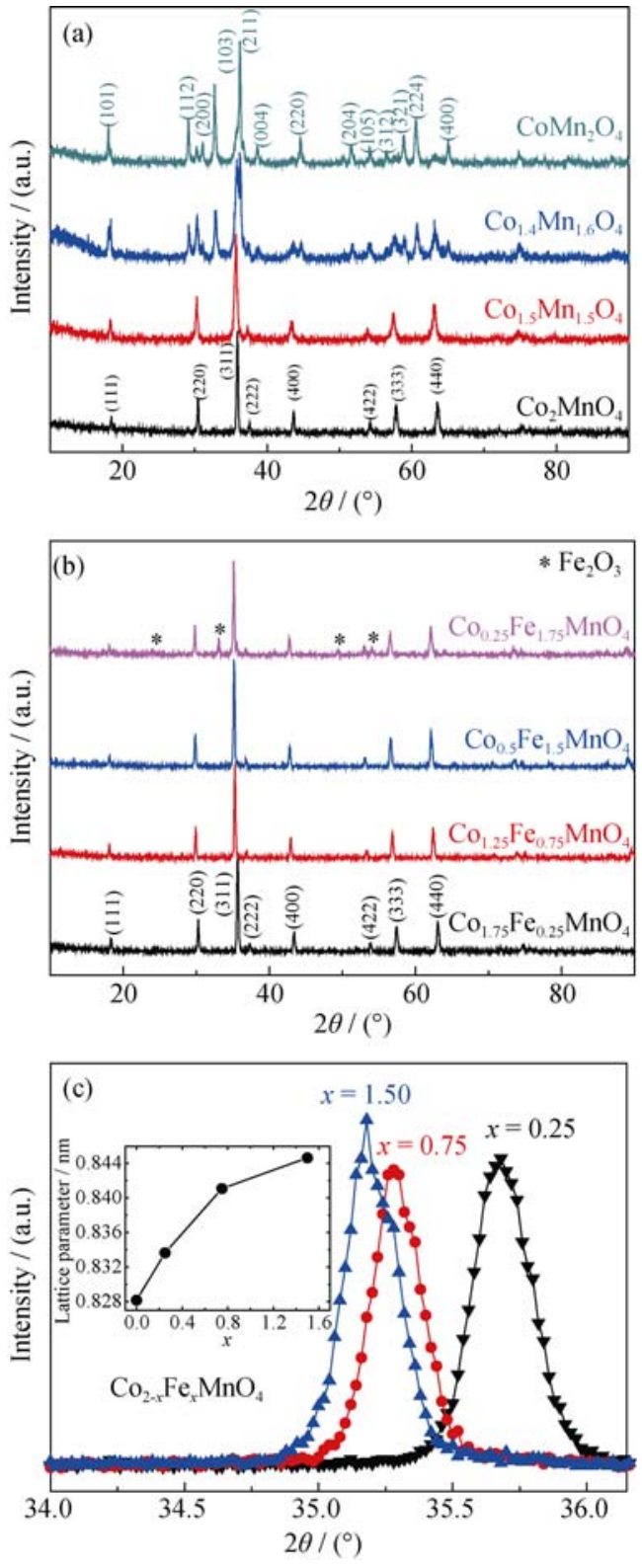

图 1 (a) $\mathrm{Co}_{2-x} \mathrm{Mn}_{1+x} \mathrm{O}_{4}(x=0,0.5,0.6,1)$ 的 XRD 图谱; (b) $\mathrm{Co}_{2-x} \mathrm{Fe}_{x} \mathrm{MnO}_{4}(x=0.25,0.75,1.50,1.75)$ 的 XRD 图谱, 星号标 出杂相; (c) $\mathrm{Co}_{2-x} \mathrm{Fe}_{x} \mathrm{MnO}_{4}$ 系列 $x=0.25,0.75,1.50$ 三个成分的 (311)衍射峰, 插图为随着 $\mathrm{Fe}$ 含量增加晶格常数 $a$ 的变化

Fig. 1 XRD patterns for $\mathrm{Co}_{2-x} \mathrm{Mn}_{1+x} \mathrm{O}_{4}(x=0,0.5,0.6,1)$ (a); XRD patterns for $\mathrm{Co}_{2-x} \mathrm{Fe}_{x} \mathrm{MnO}_{4}(x=0.25,0.75,1.50,1.75)$, the impure phase peaks are marked with asterisks; (b) Detailed XRD patterns for $\mathrm{Co}_{2-x} \mathrm{Fe}_{x} \mathrm{MnO}_{4}(x=0.25,0.75,1.50)$ showing the peak of (311) reflection (c). Inset shows the lattice parameter as a function of $\mathrm{Fe}$ content

格常数为 $a=b=c=0.8282 \mathrm{~nm}$. 在 Mn 取代 Co 的过程 中, 取代量 $x \leqslant 0.5$ 时, 可以一直保持立方尖晶石结构, 成相较好, 晶格常数有所增大, $\mathrm{Co}_{1.5} \mathrm{Mn}_{1.5} \mathrm{O}_{4}$ 的晶格常 数为 $0.8310 \mathrm{~nm}$ 。 $\mathrm{Mn}$ 含量较多时, $\mathrm{Co}_{1.4} \mathrm{Mn}_{1.6} \mathrm{O}_{4}$ 在立方 相的基础上出现第二相的衍射峰, 经检索这些峰属于 四方结构, 随着 $\mathrm{Mn}$ 含量的增加, 四方相逐渐成为主 
导, $\mathrm{CoMn}_{2} \mathrm{O}_{4}$ 成为完全的四方结构。这是由于随着 $\mathrm{Mn}$ 含量的增加, 八面体中的 $\mathrm{Mn}^{3+}$ 含量发生变化, 受到 $\mathrm{Mn}^{3+}$ 突出的 Jahn-Teller 效应的影响, 样品的晶体结构 会发生由立方相向四方相的相变 ${ }^{[10]}$ 。图 1(a)标出了 $\mathrm{CoMn}_{2} \mathrm{O}_{4}$ 的晶面指数, 其晶格常数为 $a=b=0.5734 \mathrm{~nm}$, $c=0.9286 \mathrm{~nm}$ 。图 1(b)为 $\mathrm{Fe}$ 掺杂样品 $\mathrm{Co}_{2-x} \mathrm{Fe}_{x} \mathrm{MnO}_{4}$ 的 $\mathrm{XRD}$ 图谱, 可以发现, 这一系列样品在 $0 \leqslant x \leqslant 1.50$ 成 分范围都是单相立方结构, 峰形尖锐, 成相较好。继 续增加 $\mathrm{Fe}$ 掺杂量至 $x=1.75$, 样品出现了杂相 $\mathrm{Fe}_{2} \mathrm{O}_{3}$, 表明过量 $\mathrm{Fe}$ 掺杂会以 $\mathrm{Fe}_{2} \mathrm{O}_{3}$ 的形式析出, 而非形成其 他结构相。将 $\mathrm{Fe}$ 掺杂系列样品的主要衍射峰(311)放 大, 如图 1(c)所示, 随着 $x$ 值增大, $2 \theta$ 值逐渐减小, $\mathrm{Co}_{0.5} \mathrm{Fe}_{1.5} \mathrm{MnO}_{4}$ 的峰形发生改变, 右侧有出现双峰的 趋势, 表明即将析出 $\mathrm{Fe}_{2} \mathrm{O}_{3}$ 。图 1(c)插图为掺杂 $\mathrm{Fe}$ 样 品的晶格常数随掺杂量的变化, 呈现一致上升的趋势, 这与 $\mathrm{Mn}$ 取代系列得到的结果相近, 晶格膨胀主要是 由于 $\mathrm{Fe}$ 和 $\mathrm{Mn}$ 的原子半径(0.126、0.135 nm)大于 $\mathrm{Co}$ 的原子半径 $(0.125 \mathrm{~nm})$. 但我们注意到, 随着 $\mathrm{Fe}$ 掺杂 量的增加, 晶格常数并不是线性提高, 而是呈现先快 后慢的上升趋势, 这说明影响材料晶格常数的因素虽 然以原子半径为主, 但并不是唯一的。

图 2 为室温下 $\mathrm{Co}_{2} \mathrm{MnO}_{4} 、 \mathrm{Co}_{1.7} \mathrm{Fe}_{0.3} \mathrm{MnO}_{4}$ 和 $\mathrm{CoMn}_{2} \mathrm{O}_{4}$ 样品在 $400 \sim 950 \mathrm{~cm}^{-1}$ 范围的红外光谱图, 已做归一化处理。图 2 的插图中显示了较高频范围 的主要吸收峰, $3400 \mathrm{~cm}^{-1}$ 附近的宽吸收峰为 $-\mathrm{OH}$ 吸收峰, 来自于烧结样品中存在的吸附水; 2825 2980 $\mathrm{cm}^{-1}$ 之间的双峰为 $\mathrm{C}-\mathrm{H}$ 振动峰, 来自于 空气中微量的有机物 ${ }^{[17-18]} ; 2300 \mathrm{~cm}^{-1}$ 左右的峰为空 气中的 $\mathrm{CO}_{2}$ 所引起; 在 1640 和 $1384 \mathrm{~cm}^{-1}$ 处较为明显 的吸收峰归属于未完全分解的柠檬酸盐。

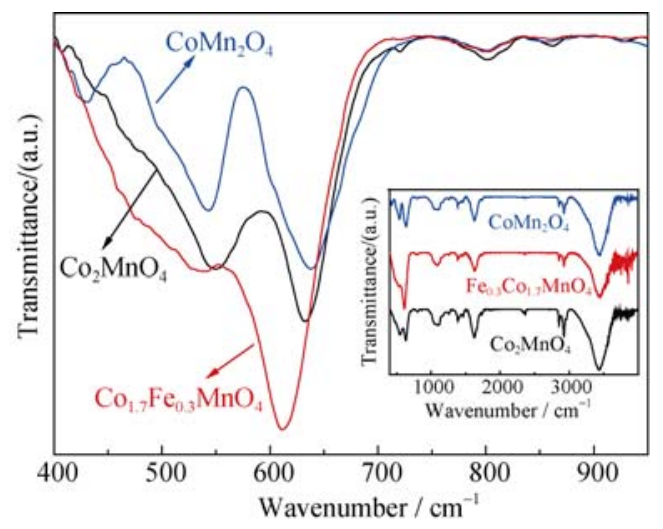

图 $2 \mathrm{Co}_{2} \mathrm{MnO}_{4} 、 \mathrm{Co}_{1.7} \mathrm{Fe}_{0.3} \mathrm{MnO}_{4}$ 和 $\mathrm{CoMn}_{2} \mathrm{O}_{4}$ 在 $400 \sim 950 \mathrm{~cm}^{-1}$ 范围的红外光谱图

Fig. 2 FT-IR spectra of $\mathrm{Co}_{2} \mathrm{MnO}_{4}, \mathrm{Co}_{1.7} \mathrm{Fe}_{0.3} \mathrm{MnO}_{4}$ and $\mathrm{CoMn}_{2} \mathrm{O}_{4}$ samples tested in $400-950 \mathrm{~cm}^{-1}$ Inset shows the testing range of $400-4000 \mathrm{~cm}^{-1}$
在 400 700 $\mathrm{cm}^{-1}$ 之间尖锐的吸收峰为金属一氧 特征振动峰 ${ }^{[18]}$ 。 $\mathrm{Co}_{2} \mathrm{MnO}_{4}$ 样品在这个范围主要有 633 和 $552 \mathrm{~cm}^{-1}$ 两个峰, 其中 $633 \mathrm{~cm}^{-1}$ 主要来自于氧 四面体位置的金属-氧离子振动, $552 \mathrm{~cm}^{-1}$ 主要来自 于氧八面体的伸缩振动, 表明样品主体为立方尖晶 石结构 ${ }^{[16,19-21]}$ 。掺杂 $\mathrm{Fe}$ 元素后, $\mathrm{Co}_{1.7} \mathrm{Fe}_{0.3} \mathrm{MnO}_{4}$ 样品 的吸收峰和 $\mathrm{Co}_{2} \mathrm{MnO}_{4}$ 比较, 向低频方向移动, 这主 要是因为 $\mathrm{Fe}$ 取代 $\mathrm{Co}$ 的过程中成键种类发生了变化, 并在一定程度上影响相邻化学键的强度和键能, 使 振动情况发生改变 ${ }^{[22]}$ 。此外, 掺杂 $\mathrm{Fe}$ 后, $552 \mathrm{~cm}^{-1}$ 左右的峰形有所展宽，这是由于 $\mathrm{Fe}$ 原子倾向于优先 占据氧八面体位置, 这个位置的成键种类在原来的 $\mathrm{Co}-\mathrm{O}$ 键基础上增加了 $\mathrm{Fe}-\mathrm{O}$ 键的共同作用，造成了 吸收峰的展宽 ${ }^{[23]}$ 。而 $\mathrm{CoMn}_{2} \mathrm{O}_{4}$ 成分由于晶体结构由 立方变成四方，金属一氧离子的成键关系更为复杂， 因此, 出现了 $432 \mathrm{~cm}^{-1}$ 的吸收峰, 和 XRD 分析结果 一致。

图 3 分别为 $5 \mathrm{~K}$ 下 $\mathrm{Co}_{2-x} \mathrm{Mn}_{1+x} \mathrm{O}_{4}$ 和 $\mathrm{Co}_{2-x} \mathrm{Fe}_{x} \mathrm{MnO}_{4}$ 系列样品的 $\mathrm{MH}$ 曲线, 插图为室温测量结果。如图 3(a)所示, $\mathrm{Co}_{2} \mathrm{MnO}_{4}$ 的饱和磁化强度为 $13.23 \mathrm{~A} \cdot \mathrm{m}^{2} / \mathrm{kg}$, 与文献[6,9]报道相近. 四方结构 $\mathrm{CoMn}_{2} \mathrm{O}_{4}$ 的饱和磁 化强度较低, 主要是由于相对于立方结构, 四方结 构对称性下降，原子间距及成键角度都发生变化， 磁性交换作用减弱，每个原子磁矩下降; 同时 $\mathrm{CoMn}_{2} \mathrm{O}_{4}$ 的 $\mathrm{MH}$ 曲线在 $7 \mathrm{~T}$ 磁场中还未饱和, 显示 出明显的磁矩非共线的特征 ${ }^{[24-26]}$, 这也是导致整体 磁性下降的原因。

表 1 总结了 $5 \mathrm{~K}$ 下两个系列样品的磁性参数随 成分的变化，可以发现，随着 $\mathrm{Mn}$ 或 $\mathrm{Fe}$ 掺杂量的增 加, 样品的饱和磁化强度都有所提高, 并且 $\mathrm{Fe}$ 掺杂 量 $x>1.0$ 之后, 矫顽力有明显的增大。

为了分析材料的磁性特征以及微观磁结构, 进 行了第一性原理计算，得到材料的分子磁矩以及每 个位置的原子磁矩。由于实际样品中的原子存在混 乱占位的现象, 并且原子磁矩并不严格共线 ${ }^{[25-26]}$, 计算所得分子磁矩与实验值存在差距，只能做定性 分析, 根据计算结果做局部磁结构示意图, 如图 4 所示。材料的磁性主要由过渡族金属原子提供, 对 于 $\mathrm{Co}_{2} \mathrm{MnO}_{4}$ 成分，材料总体呈亚铁磁结构：处于八 面体位置的 $\mathrm{Co}$ 和 $\mathrm{Mn}$ 离子磁矩是平行排列的, 而处 于四面体位置的 Co 离子磁矩与二者方向相反。这 与文献[9]结果一致。 $\mathrm{Mn}$ 与 $\mathrm{Fe}$ 原子取代 $\mathrm{Co}$ 时, 均 倾向于占据到氧八面体间隙的位置(计算所得体系 能量更低), 附近磁矩分布如图 4(b)和 4(c)所示, 处 于四面体位置的 Co 以及八面体位置的 $\mathrm{Mn}$ 原子 

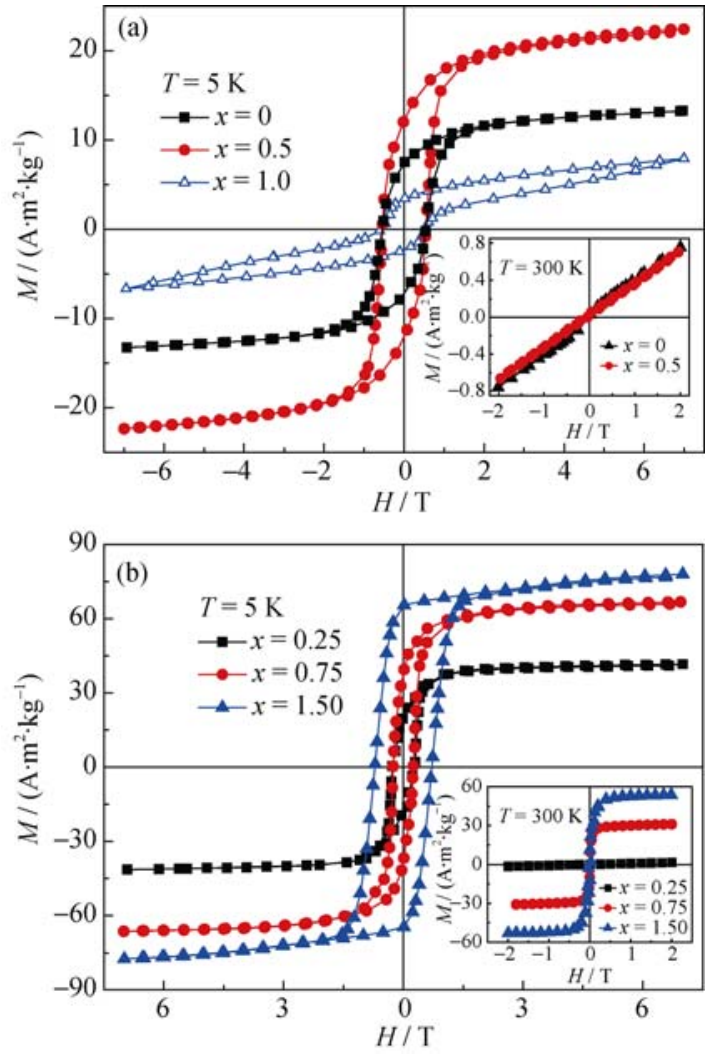

图 3 (a) $\mathrm{Co}_{2-x} \mathrm{Mn}_{1+x} \mathrm{O}_{4}(x=0,0.5,1.0)$ 于 $5 \mathrm{~K}$ 温度下的磁滞回 线, 插图为室温测量曲线; (b) $\mathrm{Co}_{2-x} \mathrm{Fe}_{x} \mathrm{MnO}_{4}(x=0.25,0.75,1.50)$ 于 $5 \mathrm{~K}$ 温度下磁滞回线, 插图为同成分室温结果

Fig. 3 Hysteresis loop for $\mathrm{Co}_{2-x} \mathrm{Mn}_{1+x} \mathrm{O}_{4}(x=0,0.5,1.0)$ (a) and $\mathrm{Co}_{2-x} \mathrm{Fe}_{x} \mathrm{MnO}_{4}(x=0.25,0.75,1.50)(\mathrm{b})$ at the temperature of $5 \mathrm{~K}$. Inset of them are the loops tested at room temperature

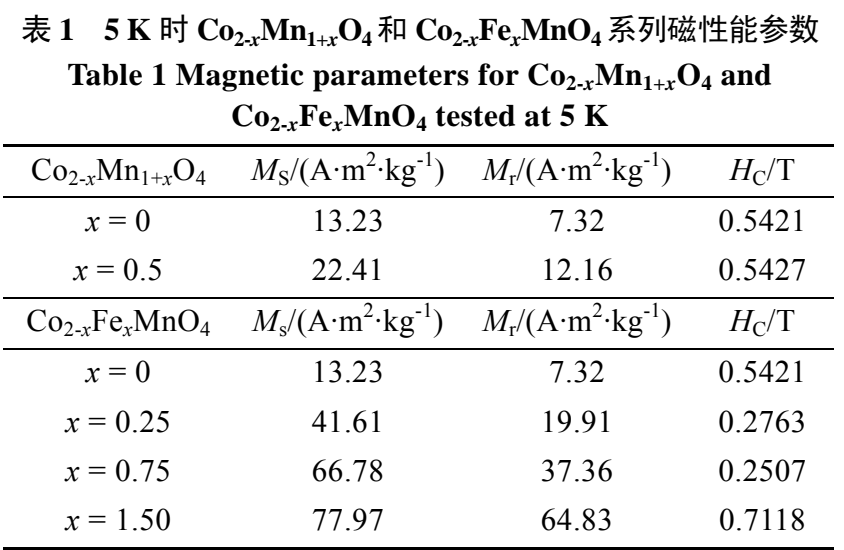

磁矩大小和方向几乎不变, 新加入的 $\mathrm{Mn} / \mathrm{Fe}$ 原子与 原来的 $\mathrm{Co}$ 原子磁矩方向相同, 只是净磁矩增大, 整 体保持了 $\mathrm{Co}_{2} \mathrm{MnO}_{4}$ 的磁结构。由于加入净磁矩更大 的原子, 掺杂原子与原有的 $\mathrm{Mn}$ (八面体)及 $\mathrm{Co}$ (四面 体)之间的磁性相互作用增强, 因此元素掺杂之后 样品的磁性有所提高。而样品的矫顽力主要反映的 是样品中亚铁磁耦合作用的强度。 $\mathrm{Fe}$ 逐渐取代 $\mathrm{Co}$ 的过程中, 尤其是 $\mathrm{Fe}$ 掺杂量较多, 四面体位置也被 $\mathrm{Fe}$ 占据时, 样品中的磁性相互作用快速增强, 因此
矫顽力会明显提高。此外，从图 3 的插图可以发现, $\mathrm{Co}_{2-x} \mathrm{Mn}_{1+x} \mathrm{O}_{4}$ 系列室温下呈顺磁状态(居里温度在室 温以下), 而 $\mathrm{Fe}$ 掺杂量较大的 $\mathrm{Co}_{2-x} \mathrm{Fe}_{x} \mathrm{MnO}_{4}$ 系列样 品在 $300 \mathrm{~K}$ 仍保持磁性特征，这说明掺杂 $\mathrm{Fe}$ 对体系 磁性耦合作用的增强作用更明显，使磁性转变温度 提高到室温以上。

居里温度为材料的磁性转变温度，通常可以间 接反映材料内部磁性耦合作用的强度, 实验测试了 $\mathrm{Co}_{2-x} \mathrm{Mn}_{1+x} \mathrm{O}_{4}(x=0,0.5,1.0)$ 样品的居里温度, 如图 5 所示, 测试场强为 $1 \mathrm{kGs}$ 。由图 5 可以发现, $\mathrm{Co}_{2} \mathrm{MnO}_{4}$ 的居里温度约为 $182 \mathrm{~K}$, 和文献[9]相近。 $x=0.5$ 时, 样 品的居里温度提高到了 $192 \mathrm{~K}$, 而 $x=1.0$ 时, 样品居 里温度为 $187 \mathrm{~K}$ 。居里温度的变化规律与图 3 (a)
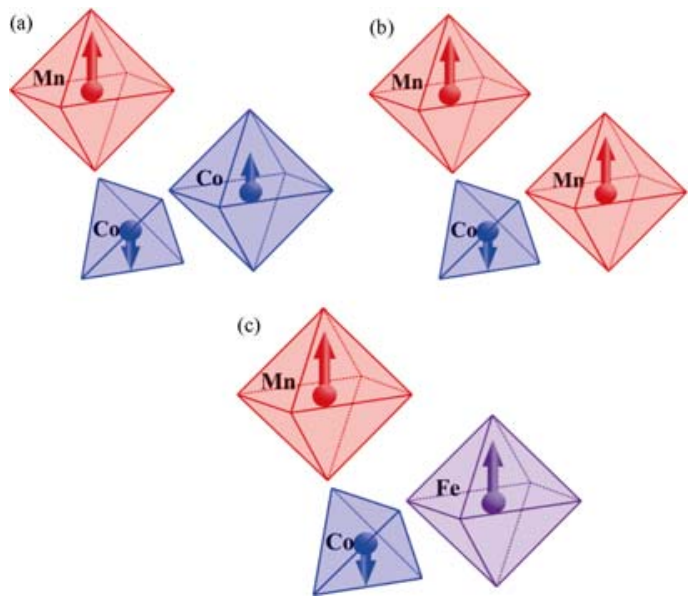

图 4 (a) $\mathrm{Co}_{2} \mathrm{MnO}_{4}$ 成分氧四面体及八面体内局部磁结构示意 图; (b) $\mathrm{Co}_{2-x} \mathrm{Mn}_{1+x} \mathrm{O}_{4}$ 中掺杂的 $\mathrm{Mn}$ 原子附近磁结构; (c) $\mathrm{Co}_{2-x} \mathrm{Fe}_{x} \mathrm{MnO}_{4}$ 中掺杂的 $\mathrm{Fe}$ 原子附近磁结构

Fig. 4 Schematic of magnetic structure for $\mathrm{Co}_{2} \mathrm{MnO}_{4}$ (a); magnetic structure near the doped atoms for $\mathrm{Co}_{2-x} \mathrm{Mn}_{1+x} \mathrm{O}_{4}$ (b) and $\mathrm{Co}_{2-x} \mathrm{Fe}_{x} \mathrm{MnO}_{4}(\mathrm{c})$

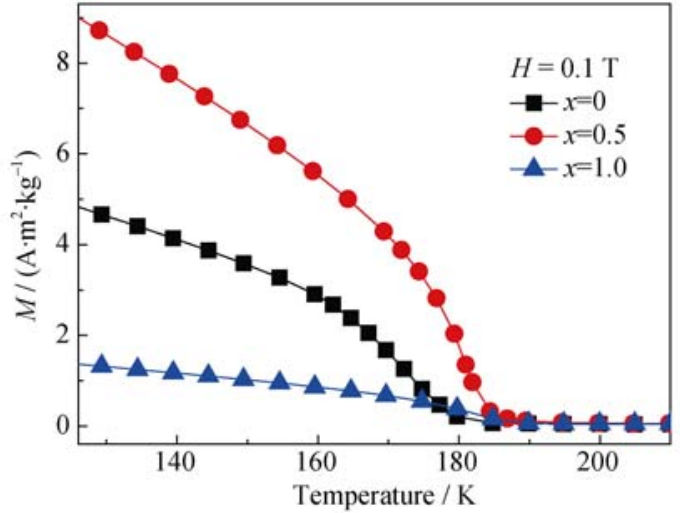

图 $5 \mathrm{Co}_{2-x} \mathrm{Mn}_{1+x} \mathrm{O}_{4}(x=0,0.5,1.0)$ 磁性随温度的变化, 测试场 强为 $0.1 \mathrm{~T}$

Fig. 5 Temperature dependence of magnetization for $\mathrm{Co}_{2-x} \mathrm{Mn}_{1+x} \mathrm{O}_{4}(x=0,0.5,1.0)$ with the field of $0.1 \mathrm{~T}$ 
的磁性规律相近: 立方结构的 $\mathrm{Co}_{2-\mathrm{x}} \mathrm{Mn}_{1+x} \mathrm{O}_{4}$ 样品随着 $\mathrm{Mn}$ 含量的增加磁性耦合作用加强, 因此饱和磁化强 度和居里温度都有所提高。而转变成四方结构之后, 原子相对位置的变化导致磁性交换作用减弱, 因此, $\mathrm{CoMn}_{2} \mathrm{O}_{4}$ 的饱和磁化强度和居里温度同时下降。

$\mathrm{Co}_{2-x} \mathrm{Fe}_{x} \mathrm{MnO}_{4}$ 系列的晶格常数随 $\mathrm{Fe}$ 含量的提 高并不是线性的, 这点与该系列分子磁矩的变化相 似, 图 6 是 $\mathrm{Co}_{2-x} \mathrm{Fe}_{x} \mathrm{MnO}_{4}$ 样品的晶格常数(Lattice parameter)和室温饱和磁化强度 $\left(M_{\mathrm{S}}\right)$ 随成分的变化, 从图中可以看出, 样品的晶格常数与饱和磁化强度 随 $x$ 值增大均呈现单调上升的趋势, 在 $x=1$ 附近出 现明显拐点, 之后上升速率变缓。如前所述, $x \leqslant 1$ 时 $\mathrm{Fe}$ 倾向于取代氧八面体位置的 $\mathrm{Co}$ 原子, $x>1$ 时 $\mathrm{Fe}$ 主要占据氧四面体。磁化强度出现拐点是由于在 $x=1$ 前后材料内的磁性相互作用不同, $x \leqslant 1$ 时八面 体位置的成键逐渐由 $\mathrm{Co}-\mathrm{O}$ 键主导变为 $\mathrm{Fe}-\mathrm{O}$ 键主 导; $x>1$ 时变化主要发生在氧四面体上。磁性作用 以及金属一氧键的改变都会影响到晶体结构, 因此 晶格常数随成分的变化也不是线性的。也就是说, 晶格常数的变化不仅仅取决于原子半径，原子间的 相互作用也有很大的影响。

\section{3 结论}

采用溶胶-凝胶法制备出 $\mathrm{Co}_{2-x} \mathrm{Mn}_{1+x} \mathrm{O}_{4}$ 和 $\mathrm{Co}_{2-x} \mathrm{Fe}_{x} \mathrm{MnO}_{4}$ 两个系列的样品, XRD 和 FT-IR 的结果 显示，金属掺杂量较少时，两个系列均保持立方结 构, 晶格常数有所增大; $\mathrm{Mn}$ 掺杂量增加到 $x \geqslant 0.6$ 时, 样品逐渐向四方结构转化, $\mathrm{Fe}$ 掺杂量 $x \geqslant 1.75$ 时出现 杂相。测量磁性发现, 立方结构样品在 $5 \mathrm{~K}$ 下显示出 铁磁特征, 随着 $\mathrm{Mn}$ 或 $\mathrm{Fe}$ 的掺入, 磁性有所提高, 主

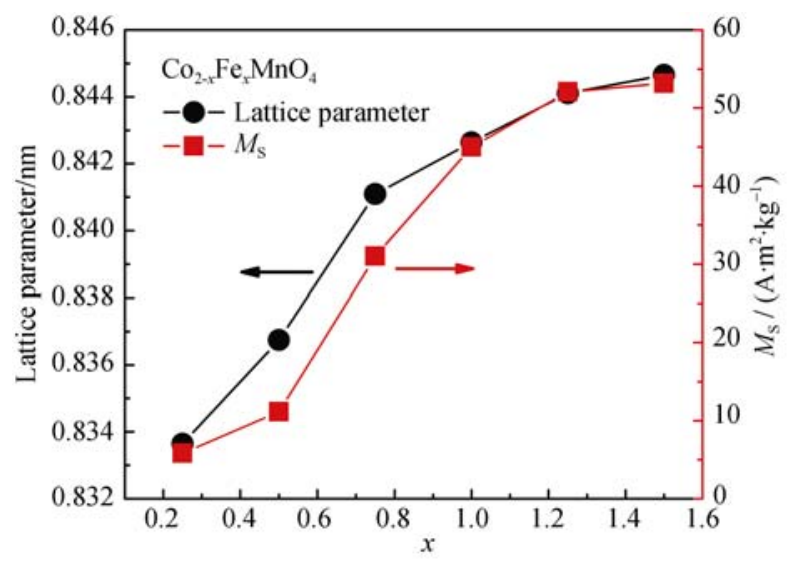

图 $6 \mathrm{Co}_{2-x} \mathrm{Fe}_{x} \mathrm{MnO}_{4}$ 样品中晶格常数(Lattice parameter)和室温 饱和磁化强度 $\left(M_{\mathrm{S}}\right)$ 随 $x$ 值的变化

Fig. 6 Lattice parameter and $M_{\mathrm{S}}$ as a function of composition
要是由于新掺入原子磁矩较大; 而掺入的 $\mathrm{Fe}$ 还能明 显提高样品的矫顽力及磁性转变温度, 说明 $\mathrm{Fe}$ 元素 在尖晶石结构中具有较强的磁性耦合作用。四方晶 体结构样品的磁性较弱。分析磁性和晶格常数规律 可以发现，晶格常数受原子半径和材料内部相互作 用的影响。

\section{参考文献:}

[1] JIN F H, KNEZ M, SCHOLZ R, et al. Monocrystalline spinel nanotube fabrication based on the Kirkendall effect. Nature Materials, 2006, 5(8): 627-631.

[2] LIANG Y, WANG H, ZHOU J, et al. Covalent hybrid of spinel manganese-cobalt oxide and graphene as advanced oxygen reduction electrocatalysts. Journal of the American Chemical Society, 2012, 134(7): 3517-3523.

[3] CHENG F Y, SHEN J, PENG B, et al. Rapid room-temperature synthesis of nanocrystalline spinels as oxygen reduction and evolution electrocatalysts. Nature Chemistry, 2011, 3(1): 79-84.

[4] YAMASAKI Y, MIYASAKA S, KANEKO Y, et al. Magnetic reversal of the FerrGslectric polarization in a multiferroic spinel oxide. Physical Review Letters, 2006, 96(20): 207204.

[5] YU L, ZHANG L, WU H B, et al. Controlled synthesis of hierarchical $\mathrm{Co}_{x} \mathrm{Mn}_{3-x} \mathrm{O}_{4}$ array micro-/nanostructures with tunable morphology and composition as integrated electrodes for lithium-ion batteries. Energy \& Environmental Science, 2013, 6(9): 2664-2671.

[6] RAJEEVAN N E, KUMAR R, SHUKLA D K, et al. Structural, electrical and magnetic properties of Bi-substituted $\mathrm{Co}_{2} \mathrm{MnO}_{4}$. Materials Science and Engineering: B, 2009, 163(1): 48-56.

[7] ROUSSET A. Reactivity of solids and new metastable phases: examples of mixed valence defect spinel ferrites and manganites. Solid State Ionics, 1994, 25(11): 236-242.

[8] LAARJ M, KACIM S, GILlOT B, et al. Cationic distribution and oxidation mechanism of trivalent manganese ions in submicrometer $\mathrm{M}_{\mathrm{nx}} \mathrm{CoFe}_{2-x} \mathrm{O}_{4}$ spinel ferrites. Journal of Solid State Chemistry, 1996, 125(1): 67-74.

[9] DOS SANTOS M E, FERREIRA R A, LISBOA-FILHO P N, et al. Cation distribution and magnetic characterization of the multiferroic cobalt manganese $\mathrm{Co}_{2} \mathrm{MnO}_{4}$ spinel doped with bismuth. Journal of Magnetism and Magnetic Materials, 2013, 329(3): 53-58.

[10] KANG S H, KIM I W, JEONG Y H, et al. Crystal growth and magnetic properties of spinel $(\mathrm{Co}, \mathrm{Mn})_{3} \mathrm{O}_{4}$. Journal of Crystal Growth, 2012, 344(1): 65-68.

[11] RAJEEVAN N E, PRADYUMNAN P P, KUMAR R, et al. MagnetGslectric properties of $\mathrm{Bi}_{x} \mathrm{Co}_{2-x} \mathrm{MnO}_{4}(0 \leqslant x \leqslant 0.3)$. Applied Physics Letters, 2008, 92(10): 102910. 
[12] JING M J, HOU H S, YANG Y C, et al. Electrochemically alternating voltage tuned $\mathrm{Co}_{2} \mathrm{MnO}_{4} / \mathrm{Co}$ hydroxide chloride for an asymmetric supercapacitor. Electrochimica Acta, 2015, 165: 198-205.

[13] HE H Q, ZHANG L, BABAEI A, et al. $\mathrm{Co}_{2} \mathrm{MnO}_{4}$ spinel-palladium co-infiltrated $\mathrm{La}_{0.7} \mathrm{Ca}_{0.3} \mathrm{Cr}_{0.5} \mathrm{Mn}_{0.5} \mathrm{O}_{3-\delta}$ cathodes for intermediate temperature solid oxide fuel cells. Journal of Alloys and Compounds, 2011, 509(40): 9708-9717.

[14] RAJEEVAN $\mathrm{N}$ E, RAVI K, SHUKLA D $\mathrm{K}$, et al. Bi-substitution-induced magnetic moment distribution in spinel $\mathrm{Bi}_{x} \mathrm{Co}_{2-x} \mathrm{MnO}_{4}$ multiferroic. Journal of Physics: Condensed Matter, 2009, 21(40): 3173-3178.

[15] RIOS E, LARA P, SERAFINI D, et al. Synthesis and characterization of manganese- cobalt solid solutions prepared at low temperature. Journal of the Chilean Chemical Society, 2010, 55(2): 261-265.

[16] HAKIM M A, KUMAR N S, SIKDER S S, et al. Cation distribution and electromagnetic properties of spinel type $\mathrm{Ni}-\mathrm{Cd}$ ferrites. Journal of Physics and Chemistry of Solids, 2013, 74(9): 1316-1321.

[17] BENNER J, THOLKAPPIYAN R, VISHISTA K, et al. Attestation in self-propagating combustion approach of spinel $\mathrm{AFe}_{2} \mathrm{O}_{4}(\mathrm{~A}=\mathrm{Co}$, $\mathrm{Mg}$ and $\mathrm{Mn}$ ) complexes bearing mixed oxidation states: Magnetostructural properties. Applied Surface Science, 2016, 383: 113-125.

[18] MANIKANDAN A, DURKA M, AMUTHA S M, et al. Sesamum indicum plant extracted microwave combustion synthesis and opto-magnetic properties of spinel $\mathrm{Mn}_{x} \mathrm{Co}_{1-x} \mathrm{Al}_{2} \mathrm{O}_{4}$ nano-catalysts. Journal of Nanoscience and Nanotechnology, 2016, 16(1): 448-456.

[19] 彭文世, 刘高魁. 矿物红外光谱图集. 贵阳: 科学出版社, 1982: 98-104.

[20] KURTAN U, GÜNGÜNEŞ H, SÖZERI H, et al. Synthesis and characterization of monodisperse $\mathrm{NiFe}_{2} \mathrm{O}_{4}$ nanoparticles. Ceramics International, 2016, 42(7): 7987-7992.

[21] 张 伟. 尖晶石型铁酸锰材料制备及性能研究. 南京: 南京理工 大学硕士学位论文, 2010.

[22] 雷中伟. 过渡金属氧化物系列红外辐射材料研究. 苏州: 苏州 大学硕士学位论文, 2007.

[23] WU C, WANG Z X, WU F, et al. Spectroscopic studies on cation-doped spinel $\mathrm{LiMn}_{2} \mathrm{O}_{4}$ for lithium ion batteries. Solid State Ionics, 2001, 144(3/4): 277-285.

[24] ZHANG S T, LU M H, WU D, et al. Larger polarization and weak ferromagnetism in quenched $\mathrm{BiFeO}_{3}$ ceramics with a distorted rhombohedral crystal structure. Applied Physics Letters, 2005, 87(26): 262907-1-3.

[25] RAJEEVAN N E, KUMAR R, SHUKLA D K, et al. MagnetGslectric behavior of ferrimagnetic $\mathrm{Bi}_{x} \mathrm{Co}_{2-x} \mathrm{MnO}_{4}(x=0,0.1$ and 0.3) thin films. Journal of Magnetism and Magnetic Materials, 2011, 323(13): 1760-1765.

[26] ZHANG H G, WANG Z, LIU E K, et al. Site preference and compensation behavior in $\mathrm{Co}(\mathrm{Cr}, \mathrm{Mn})_{2} \mathrm{O}_{4}$ system. Journal of Applied Physics, 2015, 117(17): 17B735-1-4. 